\title{
Pilot Study Examining Pregnancy-Specific Equations to Estimate Percent Body Fat in an Overweight/Obese Pregnant Hispanic Population
}

\author{
Amy Reisenberg ${ }^{1}$, Kasuen Mauldin ${ }^{1,2}$, Lisa Sawrey-Kubicek ${ }^{2}$, Mary N. R. Lesser ${ }^{2}$, and Janet \\ $\mathrm{King}^{2}$ \\ ${ }^{1}$ San José State University, San José, CA \\ ${ }^{2}$ Children's Hospital Oakland Research Institute, Oakland, CA
}

\begin{abstract}
Background and Purpose: Over half of women entering pregnancy are overweight or obese, increasing metabolic risk. This pilot study investigated whether established equations for estimating maternal percent body fat using anthropometry are accurate for Hispanic, overweight or obese pregnant women. Methods: The Siri technique of calculating percent body fat from direct measurements of body density and total body water was the gold-standard. Other pregnancy-specific equations were also examined. The study population included 15 normoglycemic, pregnant Hispanic women in their third trimester (33.2 \pm 1.9 gestational week) with a pre-pregnancy body mass index $\geq 25$ to $<40 \mathrm{~kg} / \mathrm{m}^{2}$, and with no history of chronic disease, or illicit drug, cigarette or alcohol use. Five skinfold sites, pre-pregnancy weight, current weight, and wrist circumference were measured. Body density was measured using air displacement plethysmography. Total body water was measured using ${ }^{2} \mathrm{H}_{2} \mathrm{O}$. Results: Paired t-tests showed that the Paxton equation (intended for use at gestational week 37) overestimated percent body fat compared to the Siri method, $p<0.001$, whereas the Presley equation (intended for use at gestational week 30 ) produced statistically similar results to the gold-standard, $p=0.842$. Discussion: Using skinfold thickness measurements and the Presley equation to assess percent body fat may be useful and accessible for this population.
\end{abstract}

(c) 2016 Californian Journal of Health Promotion. All rights reserved.

Keywords: pregnancy, body fat, overweight, obese, Hispanic, adiposity

\section{Introduction}

A body mass index (BMI) of $\geq 25 \mathrm{~kg} / \mathrm{m}^{2}$ is typically used in health care settings to define overweight and obesity; however the true definition of these terms is excess fat accumulation (World Health Organization [WHO], n.d.). Since the proportion of body fat can vary among individuals with the same BMI, a BMI-based approach does not accurately assess adiposity. A growing body of research strongly suggests that excess adiposity can lead to metabolic dysfunction (Ouchi, Parker, Lugus, \& Walsh, 2011), and that percent body fat $(\% \mathrm{BF})$, more than $\mathrm{BMI}$, is a better predictor of health risk (De Lorenzo et al., 2013; Frankenfield, Rowe, Cooney, Smith, \& Becker, 2001). For populations such as persons of certain ethnicities, including Hispanics, that tend to exhibit android-type obesity (characterized by central adiposity), there is even greater correlation of metabolic dysfunction due to the accumulation of visceral fat associated with central adiposity (Ouchi et al., 2011; StultsKolehmainen, Stanforth, \& Bartholomew, 2012).

The measurement of $\% \mathrm{BF}$ is not routinely performed in clinical settings, but during pregnancy, a time of significant metabolic changes, the use of $\% \mathrm{BF}$ rather than BMI to plan perinatal care could be more useful and predictive of pregnancy outcomes. In fact, maternal \%BF during pregnancy has been shown to more accurately predict perinatal outcomes than repeated measures of gestational weight gain (GWG) during pregnancy (Catalano et al., 2012; McCarthy, Strauss, Walker, \& Permezel, 2004; Suresh et al., 2012). According to the American College of Obstetricians and Gynecologists, more than half of women entering pregnancy are overweight or obese, 
placing them at higher risk for adverse perinatal and birth outcomes such as preeclampsia, neural tube defects, prematurity, caesarean delivery, large for gestational age, and subsequent insulin resistance in the child (Catalano et al., 2012; McCarthy et al., 2004; American College of Obstetricians and Gynecologists [ACOG], 2013).

In the general population, $\% \mathrm{BF}$ can be measured using dual-energy X-ray absorptiometry (DXA) or bioimpedance analysis (BIA), however these methods are not ideal in pregnancy due to radiation exposure and differences in a woman's hydration constant in a pregnant versus a nonpregnant state (Catalano, Wong, Drago, \& Amini, 1995). Many \%BF equations use assumptions for body density (BD) or a hydration constant based on a two-compartment model, which divides the body into fat and fatfree components. More accurate body fat estimates can be derived using the Siri threecompartment model (fat mass, TBW, and fatfree mass), in which BD is directly measured using hydrodensitometry or air displacement plethysmography, and TBW is measured utilizing isotopically-labeled water (Siri, 1961). $\%$ BF estimates from the Siri three-compartment model have yielded statistically similar results to the four-compartment model, which further divides the body into fat, total body water, protein, and bone mineral (Kopp-Hoolihan, Van Loan, Wong, \& King, 1999). Since bone density does not appreciably change as a result of pregnancy, in contrast to $\mathrm{TBW}$ or $\mathrm{BD}$, its measurement would not impact \%BF estimates in a pregnant population (Kopp-Hoolihan et al., 1999).

Though the above-mentioned methods are safe to use in pregnancy, they remain impractical in a typical clinical setting due to the expense, need for sophisticated equipment, and specially trained personnel. Of the various methods available to determine $\% \mathrm{BF}$, anthropometry, limited in this study to skinfold thickness, body circumference, mass and height, is the most cost-efficient and accessible method for use in the typical clinical setting. Recognizing this, two groups (Paxton et al. and Presley et al.) developed pregnancy-specific, predictive anthropometric equations to estimate body fat in healthy individuals (Paxton et al., 1998; Presley, Wong, Roman, Amini, \& Catalano, 2000). To our knowledge neither of these methods has been assessed for accuracy in a metabolically atrisk population. Thus, this pilot study aims to compare the Paxton and Presley anthropometric equations for estimating \% BF during pregnancy, against the Siri three-compartment reference model (Siri, 1961), using directly measured BD and TBW, to determine accuracy in an overweight or obese, Hispanic, pregnant population.

A secondary aim of this study was to compare the accuracy of other pregnancy-specific equations to estimate $\% \mathrm{BF}$ that have been established by Catalano et al. and Van Raajj et al. using either BD or TBW and no skinfold thickness measurements (Catalano et al., 1995; Van Raaij, Peek, Vermaat-Miedema, Schonk, \& Hautvast, 1988).

\section{Methods}

\section{Study Design}

This pilot study compared a reference method of estimating \%BF (Siri three-compartment body composition equation), against two different pregnancy-specific anthropometric methods (Paxton and Presley) using skinfolds and other easily obtained anthropometric measures (Siri, 1961; Paxton et al., 1998; Presley et al., 2000).

\section{Participants}

Fifteen pregnant women were recruited from urban hospitals and community clinics. Inclusion criteria were as follows: pregnant women between 19 and 40 years of age, selfreported pre-pregnancy BMI of $\geq 25$ to $<40$ $\mathrm{kg} / \mathrm{m}^{2}$, of Hispanic descent, singleton pregnancy, and normal glucose tolerance early in the third trimester defined as a blood glucose level of $<180 \mathrm{mg} / \mathrm{dL}$ one hour after ingesting a 75 gram glucose load (IADPSG Consensus Panel, 2010). Women who had a history of type 2 diabetes mellitus or gestational diabetes mellitus (GDM), previously delivered a large for gestational age baby, take insulin or steroids, use cigarettes, illicit drugs or alcohol, or have a chronic disease were excluded from the study. The Institutional 
Review Boards of San José State University and Children's Hospital Oakland Research Institute approved the recruitment and study protocols. All participants provided written informed consent.

\section{Measures and Procedures}

Definition of Anthropometric Measurements. Anthropometry is the "scientific study of the measurements and proportions of the human body" (English Oxford Dictionary, n.d.). For the purposes of this study, the term "anthropometric measurements" will be limited to skinfold thickness, body circumference, mass and height, as these are measurements easily obtainable in a typical clinical setting.

\section{Anthropometric Measurements.}

Anthropometric measurements, BD, and TBW were measured at the Cholesterol Research Center located in Berkeley, CA, an affiliate of the Children's Hospital Oakland Research Institute in Oakland, CA. Data for this study were collected from the women between 30 and 36 weeks of gestation.

Anthropometric measurements included body mass, height, skinfold thickness from five sites (biceps, triceps, thigh, subscapula, suprailiac), and body circumference from two sites (wrist and waist). Height was measured to the nearest $0.1 \mathrm{~cm}$ using a wall-mounted Seca 206 stadiometer. Body mass was measured using a standing digital scale that was integrated with the BOD POD by COSMED as described below.

Skinfold thickness measurements were assessed on the right side of the participant's body using Lange skinfold calipers with a constant pressure of $10 \mathrm{~g} / \mathrm{mm}^{2}$ to the nearest $0.5 \mathrm{~mm}$. Measurements were taken in duplicate and if measures differed by $>10 \%$, a third measurement of the site was taken and the average of the two closest measurements was used. The triceps measurement was a vertical skinfold, taken with the arm relaxed at the midpoint between the acromion process and the tip of the elbow. The biceps measurement was a vertical fold taken at the same midpoint on the anterior aspect of the upper arm. The subscapular measurement was an oblique skinfold taken at the lower angle of the scapula. The suprailiac measurement was an oblique skinfold taken at the iliac crest, angled down toward the pubis. The thigh measurement was a vertical skinfold taken at the midpoint of the distance between the upper border of the patella and the inguinal crease.

Body circumferences were also measured on the right side of the participant's body using a flexible, non-extensible tape with a Gulick attachment to the nearest $0.1 \mathrm{~mm}$. Wrist circumference was taken between the base of the hand and the pisiform bone. Waist circumference was taken at the widest point of the abdomen with the subject relaxed, on an exhalation.

Body Density (BD). BD was measured using air displacement plethysmography in the BOD POD (Fields, Goran, \& McCrory, 2002). The BOD POD was calibrated for atmospheric pressure prior to starting and subject information was entered into the BOD POD computing system. Participants wore minimal, tight-fitting clothing, a swim cap and a nose clip. Body mass was measured using the integrated digital scale and body volume was measured during a five-minute evaluation period during which the participant sat inside the fiberglass measurement chamber. Thoracic gas volume (TGV) was measured as a result of the subject blowing out into an attached breathing tube. Predicted TGV was used in cases where the TGV was not measurable.

Total Body Water (TBW). TBW was determined using a stable deuterium isotope and laser-based spectrophotometry technique (Murphy, 2006). Participants emptied their bladder prior to collection of a baseline saliva sample of at least $1 \mathrm{ml}$ in a Sarstedt salivette. Participants ingested a 4.5 grams dose of ${ }^{2} \mathrm{H}_{2} \mathrm{O}$ diluted in 10.5 grams of water. During the 3 hour equilibration period, subjects avoided physical activity and refrained from consuming any food or liquids. If any liquid was consumed, the volume was recorded. After the equilibration period a post ${ }^{2} \mathrm{H}_{2} \mathrm{O}$ dose saliva sample was 
collected. ${ }^{2} \mathrm{H}_{2} \mathrm{O}$ from the baseline and post-dose saliva samples were measured using a laserbased optical spectrophotometer specifically made to analyze isotopic water (Murphy, 2006).

\section{Analyses}

The Siri three-compartment body composition equation for $\% \mathrm{BF}$ was used as the reference method (Siri, 1961) with directly measured BD and TBW values for all of the participants. The two anthropometric equations used were the Paxton and Presley equations (Paxton et al., 1998; Presley et al., 2000). The six pregnancyspecific equations used to estimate \%BF using either BD or TBW and no skinfold thickness measurements were established by Catalano et al. and Van Raajj et al. (Catalano et al., 1995; Van Raaij, Peek, Vermaat-Miedema, Schonk, \& Hautvast, 1988).

The data were analyzed using Statistical Package for Social Sciences. Paired comparison $t$-tests were used to assess whether the results of various equations lacked significant difference from results given by the gold standard reference, the Siri three-compartment method. The data are expressed as mean $\pm S D$. Statistical significance was set at $p \leq 0.05$.

\section{Results}

A summary of published, pregnancy-specific equations to estimate $\% \mathrm{BF}$ are summarized in Table 1. Participant characteristics are summarized in Table 2. Anthropometric measurements were obtained at approximately $33.2 \pm 1.9$ weeks gestation. GWG at time of measurement was $6.1 \pm 4.8 \mathrm{~kg}$, which is within the recommended range of total GWG for women in the obese pre-pregnancy BMI category (Institute of Medicine [IOM], 2009).

\section{Table 1}

List of \%BF Estimation Equations: Siri Three-Compartment Reference Method and Pregnancy-Specific Equations

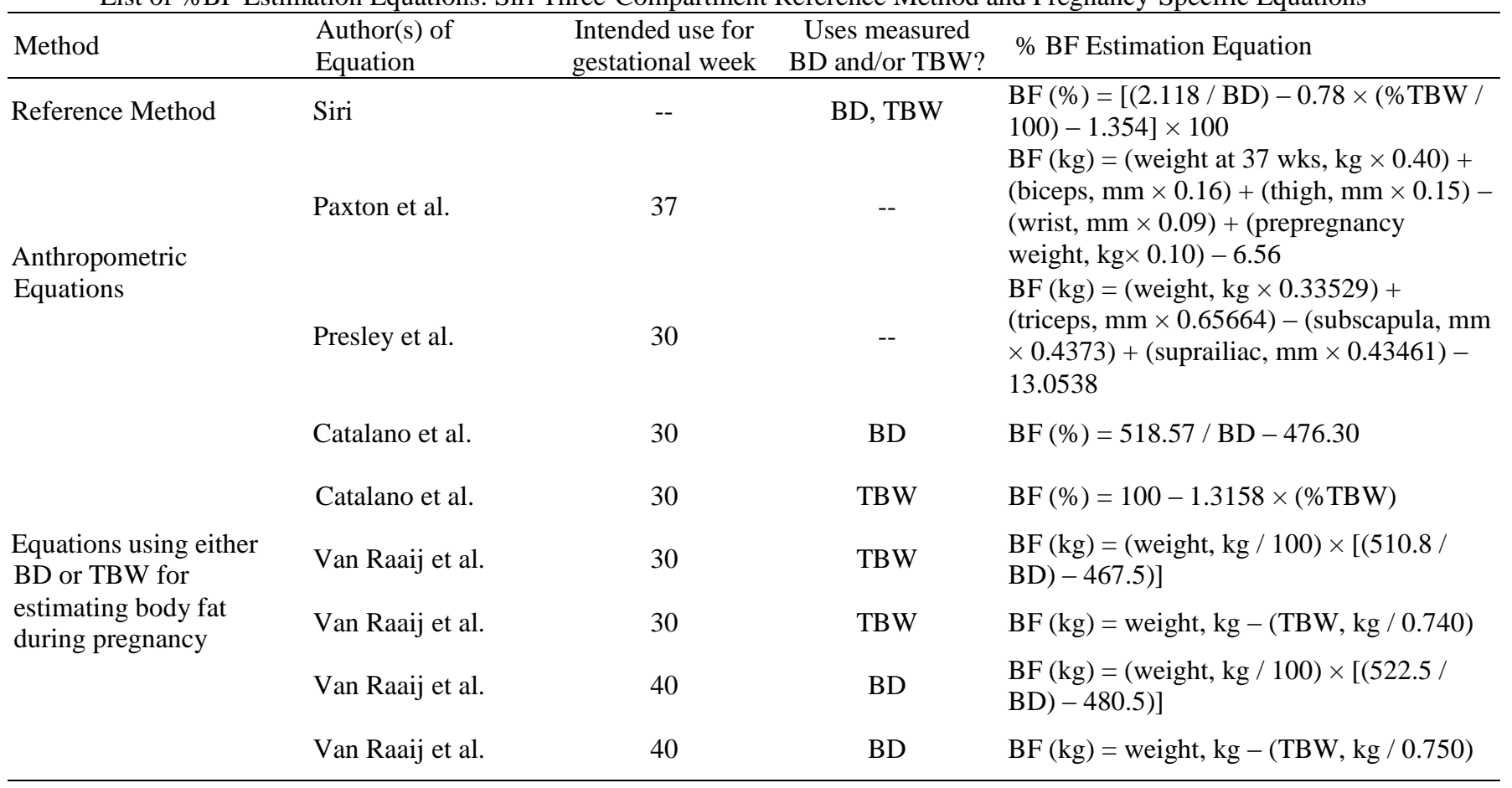

$\% \mathrm{BF}$, percent body fat; $\mathrm{BF}$, body fat; $\mathrm{BD}$, body density; TBW, total body water 
The estimated mean \%BF across all participants for the Siri three-compartment reference method and the Paxton and Presley anthropometric method skinfold equations are shown in Figure 1 and detailed in Table 3. The Siri method resulted in a mean $\pm S D \% \mathrm{BF}$ of $40.7 \% \pm 4.4 \%$. The Paxton equation resulted in a mean $\% \mathrm{BF}$ of $50.4 \% \pm 2.6 \%$, which was statistically greater when compared to results from the Siri method, $p<0.001$. The Presley equation resulted in a mean $\% \mathrm{BF}$ of $40.1 \% \pm 5.6 \%, p=0.842$, showing no statistical difference when compared to the Siri method. Note that only 14 participants were included in the Presley equation analysis, because one participant declined to have her suprailiac skinfold thickness measured, a required value in the Presley equation. There was no statistically significant correlation between GWG and \%BF (as measured by the reference Siri method) in our sample, $p=0.498$.

Figure 1.

Mean Percent Body Fat (\%BF) for Siri Three-Compartment Reference versus Paxton or Presley Anthropometric Equations

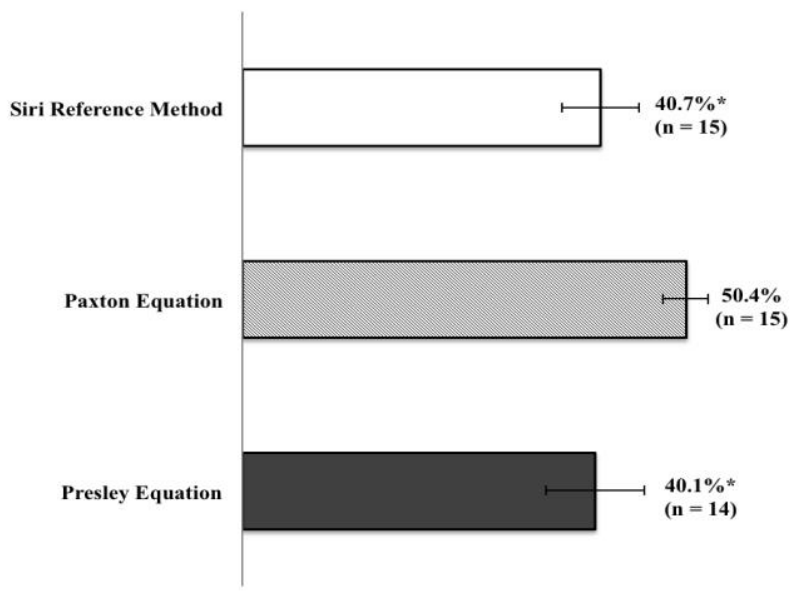

*Equation produced statistically similar results compared to Siri reference method.
Other pregnancy-specific equations to estimate $\%$ BF have been established by Catalano et al. and Van Raajj et al. using one measurement of either BD or TBW and no skinfold thickness measurements (Table 1) (Catalano et al., 1995; Van Raaij, Peek, Vermaat-Miedema, Schonk, \& Hautvast, 1988).

Table 2

\section{Characteristics of Study Participants}

\begin{tabular}{|c|c|}
\hline & $\begin{array}{c}\text { Mean } \pm \text { SD } \\
(\mathrm{n}=15)\end{array}$ \\
\hline Age (years) & $27.5 \pm 5.9$ \\
\hline Height (cm) & $157.2 \pm 7.1$ \\
\hline Pre-pregnancy weight (kg) & $81.8 \pm 14.7$ \\
\hline Pre-pregnancy BMI $\left(\mathrm{kg} / \mathrm{m}^{2}\right)$ & $32.8 \pm 4.7$ \\
\hline Gestational week & $33.2 \pm 1.9$ \\
\hline GWG $(\mathrm{kg})$ & $6.1 \pm 4.8$ \\
\hline $\begin{array}{l}\text { SD, standard deviation; } \\
\text { GWG, gestational weight ga }\end{array}$ & mass index; \\
\hline \multicolumn{2}{|c|}{$\begin{array}{l}\text { For this study, our measurements of either BD or } \\
\text { TBW value was used to estimate \%BF in our } \\
\text { study population as specified in these six } \\
\text { equations. The results from these six pregnancy- } \\
\text { specific body composition equations using BD } \\
\text { or TBW were compared against the two } \\
\text { anthropometric equations and the Siri three } \\
\text { compartment-reference method (Table 3) } \\
\text { (Catalano et al., 1995; Van Raaij et al., 1988). } \\
\text { Three of the six equations produced statistically } \\
\text { similar results to the Siri three-compartment } \\
\text { reference method ( } p \text {-values }>0.05 \text {, signifying no } \\
\text { statistical difference) (Table } 3 \text { ). }\end{array}$} \\
\hline
\end{tabular}

For this study, our measurements of either BD or TBW value was used to estimate $\% \mathrm{BF}$ in our study population as specified in these six equations. The results from these six pregnancyspecific body composition equations using BD or TBW were compared against the two anthropometric equations and the Siri three compartment-reference method (Table 3) (Catalano et al., 1995; Van Raaij et al., 1988). Three of the six equations produced statistically reference method ( $p$-values $>0.05$, signifying no statistical difference) (Table 3). 
Table 3

Comparison of Estimated Mean \%BF of Study Participants Using Various Methods

\begin{tabular}{|c|c|c|c|c|c|}
\hline Method & $\begin{array}{l}\text { Author(s) of } \\
\text { Equation }\end{array}$ & $\begin{array}{l}\text { Intended use for } \\
\text { gestational week: }\end{array}$ & $\begin{array}{l}\text { Uses measured } \\
\text { BD and/or TBW? }\end{array}$ & Mean $\% \mathrm{BF} \pm \mathrm{SD}$ & $p$ \\
\hline Reference Method & Siri & -- & $\mathrm{BD}, \mathrm{TBW}$ & $40.7 \% \pm 4.4 \%$ & -- \\
\hline \multirow{2}{*}{ Anthropometric Equations } & Paxton et al. & 37 & -- & $50.4 \% \pm 2.6 \%$ & $<0.001$ \\
\hline & Presley et al. & 30 & -- & $40.1 \% \pm 5.6 \%$ & $0.842 *$ \\
\hline \multirow{6}{*}{$\begin{array}{l}\text { Equations using either BD or TBW } \\
\text { for estimating body fat during } \\
\text { pregnancy }\end{array}$} & Catalano et al. & 30 & $\mathrm{BD}$ & $40.7 \% \pm 4.6 \%$ & $0.885^{*}$ \\
\hline & Catalano et al. & 30 & TBW & $40.8 \% \pm 4.5 \%$ & $0.564 *$ \\
\hline & Van Raaij et al. & 30 & TBW & $39.2 \% \pm 4.6 \%$ & $<0.001$ \\
\hline & Van Raaij et al. & 30 & TBW & $40.1 \% \pm 4.6 \%$ & 0.009 \\
\hline & Van Raaij et al. & 40 & $\mathrm{BD}$ & $41.8 \% \pm 4.5 \%$ & 0.003 \\
\hline & Van Raaij et al. & 40 & $\mathrm{BD}$ & $40.4 \% \pm 4.6 \%$ & $0.466 *$ \\
\hline
\end{tabular}

*Equation produced statistically similar results compared to Siri reference method

Statistical significance was set at $p<0.05$.

$\% \mathrm{BF}$, percent body fat; $\mathrm{SD}$, standard deviation; $\mathrm{BD}$, body density; TBW, total body water

\section{Discussion}

Pregnancy is a time when weight gain is socially acceptable, expected, and often in excess. Trends show that $58.8 \%$ and $55.6 \%$ of women entering pregnancy with a BMI categorized as overweight or obese, respectively, exceed the Institute of Medicine GWG recommendations (Dalenius, Brindley, Smith, Reinold, \& Grummer-Strawn, 2012). In comparison, only $38.6 \%$ of women entering pregnancy with a BMI categorized as normal exceed GWG recommendations (Dalenius et al., 2012). Total GWG and the composition of GWG can be highly variable among women. GWG is composed of water, fat, protein and minerals deposited in the fetus, placenta, amniotic fluid, uterus, mammary gland, blood, and adipose tissue (Gilmore, Klempel-Donchenko, \& Redman, 2015). The rate of weight gain tends to be higher in the second and third trimesters compared to the first (IOM, 2009). In the third trimester, much of the weight gain is typically due to fetal growth and increases in TBW (Pitkin, 1976). Fat mass accretion occurs throughout pregnancy (Pitkin, 1976). Prepregnancy body composition has influence on the amount and distribution of adipose tissue gained during pregnancy. Women who enter pregnancy obese tend to accrue fat more centrally during pregnancy compared to lean women, and this excess central fat is associated with increased risk for insulin resistance and gestational diabetes (Ehrenberg, Huston-Presley, \& Catalano, 2003).

Excess central fat accumulation is of even greater concern in our study population since overweight/obese women of Hispanic ethnicity already tend to exhibit central adiposity regardless of pregnancy (Ouchi et al., 2011; Stults-Kolehmainen et al., 2012). Given the majority of overweight and obese women have excessive GWG, having an accurate way to assess \% $\mathrm{BF}$ at the start of the third trimester in this population can help clinicians identify high- 
risk women for intensive nutrition and other lifestyle interventions.

The estimation of $\% \mathrm{BF}$ in pregnant women poses special challenges due the various physiological changes that take place in the maternal body during gestation. The standard assumption of fat free mass being $73 \%$ water (Kopp-Hoolihan et al., 1999) cannot be used during pregnancy because tissues synthesized during pregnancy, such as the placenta, can contribute to a significant portion of TBW (IOM, 2009). In the pregnant state, TBW increases and overall BD decreases, thus equations that rely upon only one measure of these two variables will over- or underestimate $\%$ BF. For this reason, the Siri threecompartment model, which relies upon direct measurement of both TBW and BD was used in this study.

These study results point to using the Presley equation for estimating $\% \mathrm{BF}$ as the Paxton equation significantly overestimated $\% \mathrm{BF}$ in this study's overweight or obese, pregnant population. The tendency of the Paxton equation to yield higher \%BF estimates is consistent with Robič et al's findings (Robič et al., 2014). Various explanations for this result should be considered. A key difference between the Paxton and Presley equations is that the Paxton equation includes pre-pregnancy weight. In this study population of women who are already overweight or obese entering into pregnancy, including pre-pregnancy weight may overestimate their \%BF, which was indeed the case for all of the study participants.

When using pregnancy-specific anthropometric equations to estimate $\% \mathrm{BF}$, one must consider gestational age as each equation is intended for use at a specific week of gestation. For example, the Presley equation is intended for use at 30 weeks gestation, while the Paxton equation, at 37 weeks. The average gestational age of participants in this study was 33.2 weeks, slightly closer to the intended gestational week of the Presley equation, which may in part explain the results. Another major difference between the two anthropometric equations is that the Presley equation uniquely uses two central skinfold sites (subscapula and suprailiac), which may more accurately reflect the pattern of central fat deposition during pregnancy thus providing a more accurate estimate of percent body fat in this overweight/obese pregnant population (Ehrenberg et al., 2003; Taggart, Holliday, Billewicz, Hytten, \& Thomson, 1967).

Catalano et. al and Van Raaij et. al developed a total of six pregnancy-specific equations to estimate \%BF based on either measured TBW or BD (Catalano et al., 1995; Van Raaij et al., 1988). It is interesting to note that two out of the three equations that yielded statistically similar results to the Siri three-compartment model, rely on direct measures of $\mathrm{BD}$ and the other relies on the direct measure of TBW. Because BD is an assessment of all body compartments versus TBW, which is included only in fat-free mass, using $\mathrm{BD}$ may lead to more accurate \% $\mathrm{BF}$ estimates.

\section{Limitations}

A limitation of this study is that the pregnancyspecific body fat equations were not necessarily used during the gestational weeks for which they were intended. Another study limitation is that skinfold assessment through use of calipers is subject to operator error. To minimize operator error in this study, training and practice sessions within the research team were held, during which skinfold measurements were taken of various peripheral and central sites. These sessions continued until consistency was reached and maintained in repeated measurements by the same operator and between operators. Two operators collected skinfold thickness measurements for this study.

\section{Conclusion}

Future research pursuits stemming from this work include extending the study population to capture other ethnic groups such as South and East Asian pregnant women who tend to have higher percent body fat than Caucasian women with the same BMI and tend to accrue fat centrally (WHO Expert Consultation, 2004). Another key area to study in the future is 
tracking the change in $\% \mathrm{BF}$ during the course of pregnancy, rather than at one point in time. This information would provide clinicians and researchers further insight into the composition of weight gain attributable to maternal fat, which in excess may have adverse metabolic consequences, versus expansion in blood volume, fetal weight, and tissue synthesis during pregnancy. For example, tracking fat gain in women with metabolic alterations during pregnancy, such as those with GDM, may be of interest in the context of metabolic programming and the predisposition of offspring of GDM mothers to develop metabolic diseases later in life (Gilmore et al., 2015). The ability to differentiate composition of pregnancy weight gain may lead to tailored clinical interventions that can mitigate adverse outcomes such as caesarean section and later risk of developing type 2 diabetes in both mother and child.

Current clinical practice focuses on tracking GWG throughout pregnancy and recommending rate of gain determined by the woman's prepregnancy BMI (IOM, 2009). The results of this study show that GWG was not correlated with $\% \mathrm{BF}$ during 30-37 weeks gestation for overweight/obese, Hispanic mothers. Thus tracking maternal $\% \mathrm{BF}$ is more useful than
GWG because excess body fat has a greater effect on metabolism and adverse pregnancy outcomes (Catalano et al., 2012; McCarthy et al., 2004; Suresh et al., 2012). Using the Presley anthropometric equation provides clinicians a useful and practical method to assess \% BF in this metabolically at-risk population of overweight/obese Hispanic women during pregnancy. Assessing \% BF may lead to more specific clinical recommendations based on a woman's body composition rather than BMI, to mitigate adverse birth outcomes. Future studies to develop anthropometric equations intended for use in early pregnancy can give clinicians the ability to identify higher-risk pregnancies earlier and a means of tracking body fat gain throughout pregnancy to have a greater impact on perinatal and long-term health.

\section{Acknowledgements}

Funding for this project was provided by the California State University Program for Education and Research in Biotechnology New Investigator Grant Program, 2012 (PI: KM), the San Jose State University Department of Nutrition, Food Science, and Packaging Circle of Friends Research Assistance Award, 2013 (AR), and the Almond Board of California (PI: $\mathrm{JK})$.

\section{References}

American College of Obstetricians and Gynecologists. (2013). ACOG Committee opinion no. 549: obesity in pregnancy. Obstetrics and Gynecology, 121(1), 213.

Catalano, P. M., McIntyre, H. D., Cruickshank, J. K., McCance, D. R., Dyer, A. R., Metzger, B. E., ... \& Persson, B. (2012). The Hyperglycemia and Adverse Pregnancy Outcome Study Associations of GDM and obesity with pregnancy outcomes. Diabetes Care, 35(4), 780-786.

Catalano, P. M., Wong, W. W., Drago, N. M., \& Amini, S. B. (1995). Estimating body composition in late gestation: a new hydration constant for body density and total body water. American Journal of Physiology-Endocrinology And Metabolism, 268(1), E153-E158.

Dalenius, K., Brindley, P., Smith, B., Reinold, C., \& Grummer-Strawn, L. (2012). Pregnancy Nutrition Surveillance 2010 Report. Atlanta: U.S. Department of Health and Human Services, Center for Disease Control and Prevention.

De Lorenzo, A., Bianchi, A., Maroni, P., Iannarelli, A., Di Daniele, N., Iacopino, L., \& Di Renzo, L. (2013). Adiposity rather than BMI determines metabolic risk. International Journal of Cardiology, 166(1), 111-117.

Ehrenberg, H. M., Huston-Presley, L., \& Catalano, P. M. (2003). The influence of obesity and gestational diabetes mellitus on accretion and the distribution of adipose tissue in pregnancy. American Journal of Obstetrics and Gynecology, 189(4), 944-948.

English Oxford Dictionary. (n.d.). Anthropometry. Available at: http://www.oxforddictionaries.com/us/definition/american english/anthropometry. Accessed August 24, 2016. 
Fields, D. A., Goran, M. I., \& McCrory, M. A. (2002). Body-composition assessment via airdisplacement plethysmography in adults and children: a review. The American Journal of Clinical Nutrition, 75(3), 453-467.

Frankenfield, D. C., Rowe, W. A., Cooney, R. N., Smith, J. S., \& Becker, D. (2001). Limits of body mass index to detect obesity and predict body composition. Nutrition, 17(1), 26-30.

Gilmore, L. A., Klempel-Donchenko, M., \& Redman, L. M. (2015, June). Pregnancy as a window to future health: Excessive gestational weight gain and obesity. In Seminars in Perinatology (Vol. 39, No. 4, pp. 296-303). WB Saunders.

IADPSG Consensus Panel. (2010) International Association of Diabetes and Pregnancy Study Groups (IADPSG) recommendations on the diagnosis and classification of hyperglycemia in pregnancy. Diabetes Care, 33(3), 676-682.

Institute of Medicine [IOM]. (2009). Weight gain during pregnancy: reexamining the guidelines. Rasmussen, K. M., \& Yaktine, A. L. (Eds.). National Academies Press.

Kopp-Hoolihan, L. E., Van Loan, M. D., Wong, W. W., \& King, J. C. (1999). Fat mass deposition during pregnancy using a four-component model. Journal of Applied Physiology, 87(1), 196-202.

McCarthy, E. A., Strauss, B. J., Walker, S. P., \& Permezel, M. (2004). Determination of maternal body composition in pregnancy and its relevance to perinatal outcomes. Obstetrical \& Gynecological Survey, 59(10), 731-742.

Murphy, E. J. (2006). Stable isotope methods for the in vivo measurement of lipogenesis and triglyceride metabolism. Journal of Animal Science, 84(13_suppl), E94-E104.

Ouchi, N., Parker, J. L., Lugus, J. J., \& Walsh, K. (2011). Adipokines in inflammation and metabolic disease. Nature Reviews Immunology, 11(2), 85-97.

Paxton, A., Lederman, S. A., Heymsfield, S. B., Wang, J., Thornton, J. C., \& Pierson, R. N. (1998). Anthropometric equations for studying body fat in pregnant women. The American Journal of Clinical Nutrition, 67(1), 104-110.

Pitkin, R.M. (1976) Nutritional support in obstetrics and gynecology. Clinical Obstetrics and Gynecology, 19(3), 489-513.

Presley, L. H., Wong, W. W., Roman, N. M., Amini, S. B., \& Catalano, P. M. (2000). Anthropometric estimation of maternal body composition in late gestation. Obstetrics \& Gynecology, 96(1), 3337.

Robič, T., Benedik, E., Fidler Mis, N., Bratanič, B., Rogelj, I., \& Golja, P. (2014). Challenges in determining body fat in pregnant women. Annals of Nutrition and Metabolism, 63(4), 341-349.

Siri, W. E. (1961). Body composition from fluid spaces and density: analysis of methods. Techniques for Measuring Body Composition, 61, 223-44.

Stults- Kolehmainen, M. A., Stanforth, P. R., \& Bartholomew, J. B. (2012). Fat in android, trunk, and peripheral regions varies by ethnicity and race in college aged women. Obesity, 20(3), 660-665.

Suresh, A., Liu, A., Poulton, A., Quinton, A., Amer, Z., Mongelli, M., ... \& Nanan, R. (2012). Comparison of maternal abdominal subcutaneous fat thickness and body mass index as markers for pregnancy outcomes: A stratified cohort study. Australian and New Zealand Journal of Obstetrics and Gynaecology, 52(5), 420-426.

Taggart, N. R., Holliday, R. M., Billewicz, W. Z., Hytten, F. E., \& Thomson, A. M. (1967). Changes in skinfolds during pregnancy. British Journal of Nutrition, 21(02), 439-451.

Van Raaij, J. M., Peek, M. E., Vermaat-Miedema, S. H., Schonk, C. M., \& Hautvast, J. G. (1988). New equations for estimating body fat mass in pregnancy from body density or total body water. The American Journal of Clinical Nutrition, 48(1), 24-29.

World Health Organization [WHO]. (n.d.). Global database on Body Mass Index. Available at: http://apps.who.int/bmi/index.jsp?introPage=intro_3.html. Accessed August 24, 2016.

World Health Organization [WHO] Expert Consultation. (2004). Appropriate body-mass index for Asian populations and its implications for policy and intervention strategies. Lancet, 363(9403), 157. 